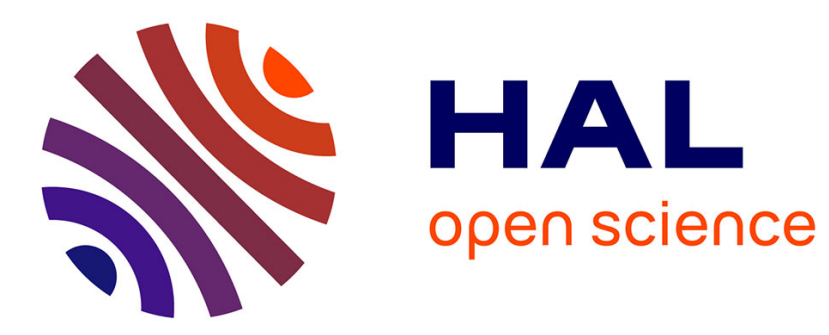

\title{
L'agglomération secondaire antique d'Optevoz (Isère). Un état des connaissances
}

Robert Royet, Catherine Coquidé

\section{To cite this version:}

Robert Royet, Catherine Coquidé. L'agglomération secondaire antique d'Optevoz (Isère). Un état des connaissances. Revue archéologique de Narbonnaise, 2005, 38 (1), pp.141-155. halshs-01966984

\section{HAL Id: halshs-01966984 \\ https://shs.hal.science/halshs-01966984}

Submitted on 30 Dec 2018

HAL is a multi-disciplinary open access archive for the deposit and dissemination of scientific research documents, whether they are published or not. The documents may come from teaching and research institutions in France or abroad, or from public or private research centers.
L'archive ouverte pluridisciplinaire HAL, est destinée au dépôt et à la diffusion de documents scientifiques de niveau recherche, publiés ou non, émanant des établissements d'enseignement et de recherche français ou étrangers, des laboratoires publics ou privés. 


\title{
L'agglomération secondaire antique d'Optevoz (Isère). Un état
} des connaissances

In: Revue archéologique de Narbonnaise, Tome 38-39, 2005. pp. 141-155.

\begin{abstract}
The multiplication of preliminary soundings in the village of Optevoz, crossed with the results obtained from systematic prospecting in the surrounding countryside, has enabled to update the vision of an antique town we still know little about in spite of ancient discoveries. This article suggests that the status of this gathered settlement was secondary to a large nearby villa.
\end{abstract}

\section{Résumé}

La multiplication de sondages préalables dans le village d'Optevoz croisés avec les résultats de prospections systématiques des campagnes environnantes permet de renouveler la vision d'une agglomération antique jusqu'ici mal connue malgré des découvertes anciennes. Cet article suggère la subordination de cet habitat groupé à une grande villa voisine.

Citer ce document / Cite this document :

Royet Robert, Coquidé Catherine. L'agglomération secondaire antique d'Optevoz (Isère). Un état des connaissances. In: Revue archéologique de Narbonnaise, Tome 38-39, 2005. pp. 141-155.

http://www.persee.fr/web/revues/home/prescript/article/ran_0557-7705_2005_num_38_1_1155 


\title{
L’agglomération secondaire antique d'Optevoz (Isère). Un état des connaissances
}

\author{
Robert ROYET et Catherine COQUIDÉ
}

\begin{abstract}
Résumé: La multiplication de sondages préalables dans le village d'Optevoz croisés avec les résultats de prospections systématiques des campagnes environnantes permet de renouveler la vision d'une agglomération antique jusqu'ici mal connue malgré des découvertes anciennes. Cet article suggère la subordination de cet habitat groupé à une grande villa voisine.

Mots-clés: Gaule Narbonnaise, cité de Vienne, Allobroges, agglomération secondaire, villa
\end{abstract}

Abstract: The multiplication of preliminary soundings in the village of Optevoz, crossed with the results obtained from systematic prospecting in the surrounding countryside, has enabled to update the vision of an antique town we still know little about in spite of ancient discoveries. This article suggests that the status of this gathered settlement was secondary to a large nearby villa.

Keywords : Gallia Narbonenesis, city of Vienna, Allobroges, secondary agglomeration, villa

Depuis le XIX'e siècle, la commune d'Optevoz, située à $45 \mathrm{~km}$ à l'est de Lyon, livre des témoins importants des périodes gauloise et gallo-romaine. Le bourg actuel luimêmc est réputé avoir succédé à une agglomération antique plus vaste. Soumis actuellement à un important accroissement démographique, le village fait donc depuis plusieurs années l'objet d'opérations d'évaluations archéologiques systématiques dont les résultats restent toutefois difficilement interprétables. Il apparaît ainsi aujourd'hui utile de synthétiser l'état des connaissances ${ }^{1}$. Cette étude vise à évaluer l'importance réelle de cette agglomération en la réintégrant dans son contexte topographique et archéologique. Outre les principaux enseignements des sondages dans le village, la carte archéologique du bassin d'Optevoz et de ses abords est aussi présentée. Malgré les lacunes de la documentation il s'agit de proposer une mise en réseau de l'agglomération avec les sites environnants, notamment la grande villa reconnue au lieu-dit "le Paradis" qui pourrait avoir exercé un rôle majeur dans cette micro-région.

\section{LOCALISATION ET CADRE ENVIRONNEMENTAL}

Optevoz est implanté au cœur de l'Isle-Crémieu, petit massif calcaire aux confins des départements de l'Isère et de l'Ain (fig. 1). Ultime piémont mériđional du Jura, ce plateau forme un triangle à peu pròs isocèle de $25 \mathrm{~km}$ de côté bordé par le Rhône sur les $2 / 3$ de son périmètre. Dressant sur son flanc occidental des falaises surplombant le fleuve de près de $200 \mathrm{~m}$, cette table calcaire s'affaisse progressivement vers le sud-est jusqu'au niveau de la vallée. Depuis la plaine, plusieurs failles (Fusa, Ry, Amby), surcreusées par des petits cours d'eau, ont naturellement servi de voies d'accès au sommet du plateau dès

1 Cet article dresse un état des connaissances disponibles au début de l'année 2004. 


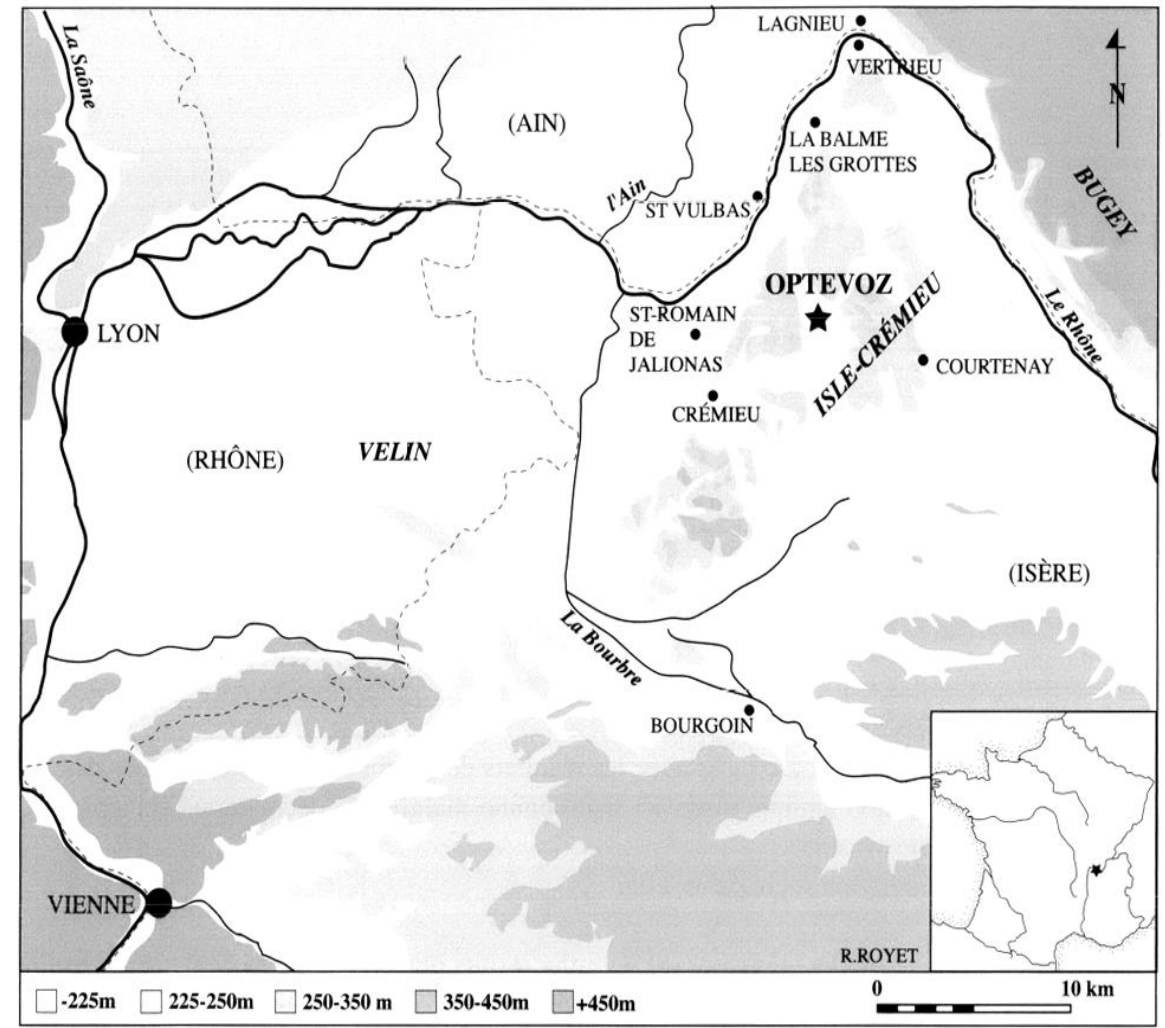

Fig. 1 Localisation de l'Isle Crémieu et d'Optevoz.
l'Antiquité (fig. 2). La dernière glaciation a profondément remodelé la surface de ce massif en déposant de nombreuses buttes morainiques et en creusant des dolines. Ces dépressions, colmatées avec des sédiments fluvio-glaciaires grossiers, concentrent l'essentiel des surfaces cultivables. A contrario la plus importante partie du plateau, où le substrat (calcaire bathonien du plateau ou galets et graviers des dépôts morainiques) affleure, est à peu près stérile.

Le village est installé au centre d'une des principales dolines, d'une surface de 300 ha, qu'il partage avec le hameau de Siccieu au sud (fig. 3 et 4). Depuis la vallée du Rhône, on accède facilement à cette plaine par le Val d'Amby qui débouche directement à son extrémité septentrionale ou, par le sud-ouest, via le chemin suivi par l'actuelle D52. Cette cuvette est bordée à l'ouest par les falaises du Mont d'Annoisin qui dressent une barrière, haute d'une centaine de mètres, dont le piémont est recouvert d'un épais bourrelet fluvio-glaciaire. Ses trois autres côtés sont délimités par un complexe de moraines flanquées de petites dépressions humides souvent aménagées en étangs au cours de ces derniers siècles. Ces réservoirs artificiels ont modifié considérablement l'hydrographie locale qui est aujourd'hui largement canalisée. La plaine est traversée sur toute sa longueur par le ruisseau dit "des Moulins" qui, en aval, devient l'Amby après avoir collecté l'eau de plusieurs sources. En complément de cette rivière, de multiples résurgences karstiques permettent de disposer, au pied des buttes et falaises, d'une eau abon- dante la plus grande partie de l'année. Ce bassin cultivé offre un contraste saisissant avec les vastes étendues au rocher affleurant où ne poussent qu'un maigre taillis ou des pelouses sèches. Pour autant, les sols fersiallitiques qui constituent la plus grande partie des terrains agricoles utiles souffrent de leur excessive perméabilité et d'une épaisseur insuffisante. Seules les dépressions offraient, après drainage, des sols épais plus propices à des cultures peu enrichies en engrais. Les scènes bucoliques peintes par les artistes qui, autour de Corot et Daubigny, constituèrent l'école d'Optevoz pendant la seconde moitié du $\mathrm{XIX}^{\mathrm{e}}$ siècle, ne doivent pas estomper l'aridité de ces lieux.

\section{HISTORIQUE DES DÉCOUVERTES}

Si on a pu prétendre - avec quelque exagération - qu' " aucun sol n'a restitué plus d'inscriptions, de statuettes, de bijoux et de médailles» (Chagny 1929), force est de constater que cette commune n'a bénéficié d'aucune recherche cohérente jusqu'à une date récente. Des découvertes fortuites d'éléments gallo-romains s'y sont toutefois multipliées dès le XIX ${ }^{\mathrm{e}} \mathrm{s}$.

La grande villa du "Paradis" (fig. 4, $\mathrm{n}^{\circ} 1$ et fig. 9) a ainsi livré deux dolia et un trésor comprenant des monnaies datées de Philippe l'Arabe, des cuillers d'argent et un collier d'or (Commarmond 1855) conservés dans une aiguière.

Un autre trésor contemporain découvert en 1834, dans un lieu indéterminé (peut être dans le village), aurait com- 
Fig. 2. Carte archéologique simplifiée de I'Isle Crémieu. 1, limites de la commune d'Optevoz; 2, bourg d'Optevoz; 3, agglomérations antiques; 4 , villa; 5 , voies antiques: a) principales, b) secondaires, c) hypothétiques.

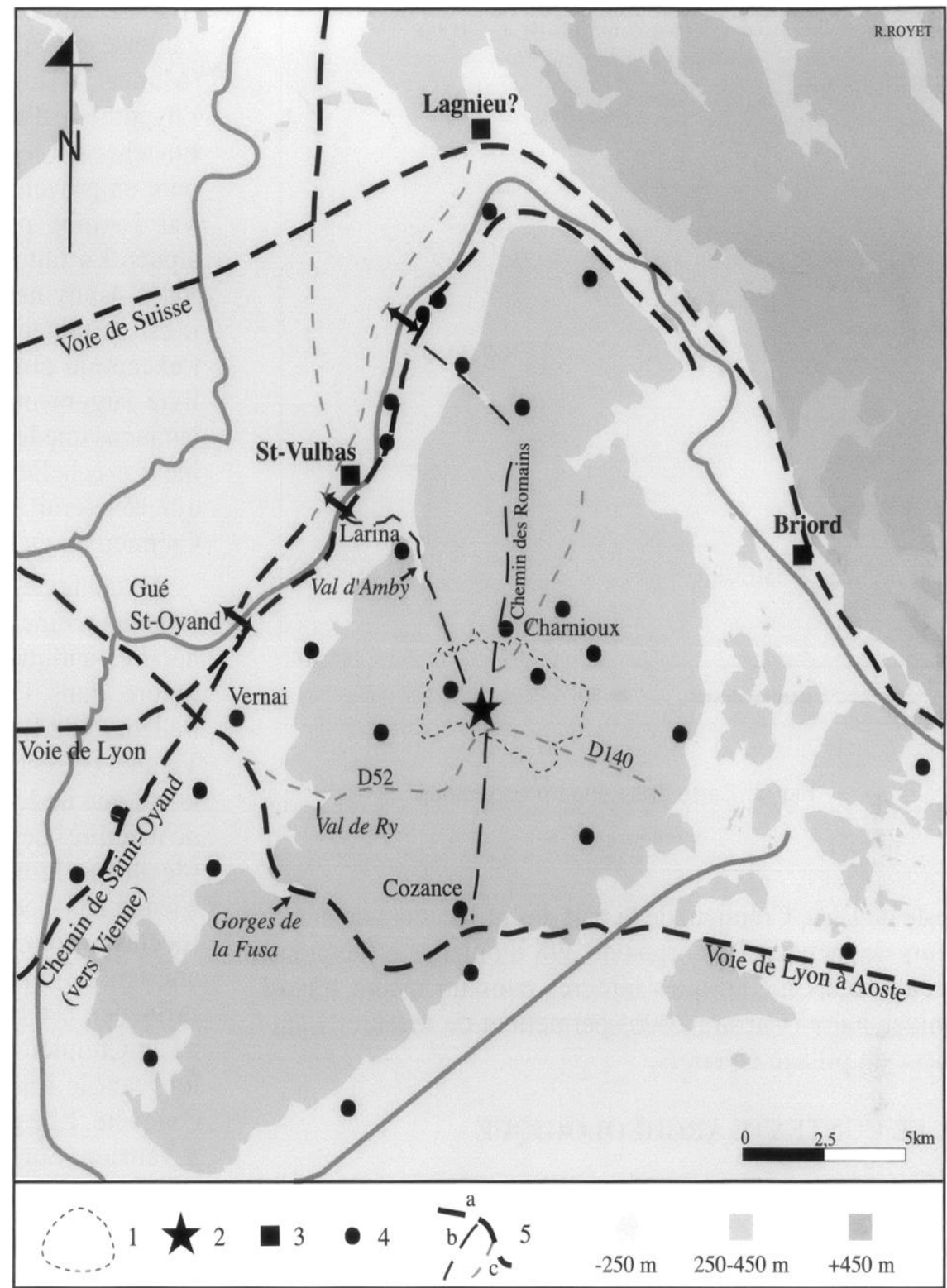

porté un nombre inconnu de monnaies, deux colliers, des anneaux d'or et d'argent ainsi que des épingles en or, un bracelet d'argent et deux cuillers d'argent (Loriot, Rémy 1988).

Une statuette de Sucellus fut trouvée en 1908 (Müller note $\mathrm{ms} \mathrm{n}^{\circ} 87$; Boucher, Tassinari 1976) en un lieu inconnu de la commune.

Des découvertes ponctuelles, notamment liées à des destructions, furent signalées par J. Chauffin sous forme de notices succinctes dans un répertoire régional (Chauffin 1960 et 1981).

Il a fallu attendre 1985 pour que soient entrepris les premiers véritables travaux de prospection sur la commune. La quasi-totalité des sites actuellement connus sur Optevoz fut signalée par R. Convert dans le cadre plus vaste d'un recensement s'étendant à toute l'Isle Crémieu. Cet inventaire resté inédit, interrompu par le décès pré- maturé de son auteur, fut repris et approfondi pour les communes d'Optevoz et de Saint-Baudille-de-la-Tour par $S$. Carrara dans le cadre d'un mémoire de maîtrise (Carrara 2000).

Sur l'agglomération antique proprement dite, les travaux de construction sont systématiquement précédés d'une évaluation archéologique depuis 1997. À ce jour, quatorze opérations ont permis d'évaluer 4,75 ha du village ou de ses environs immédiats sans qu'il ait encore été possible de réaliser une fouille extensive (fig. 5). L'efficacité de ce contrôle doit malheureusement être relativisée. Ces dernières années, plusieurs opérations d'urbanisme ont été réalisées sans respect des procédures ou des prescriptions techniques et ont détruit des vestiges, quelquefois en connaissance de cause.

Ces destructions sont aggravées par la concurrence que rencontrent les archéologues, à Optevoz comme dans le 


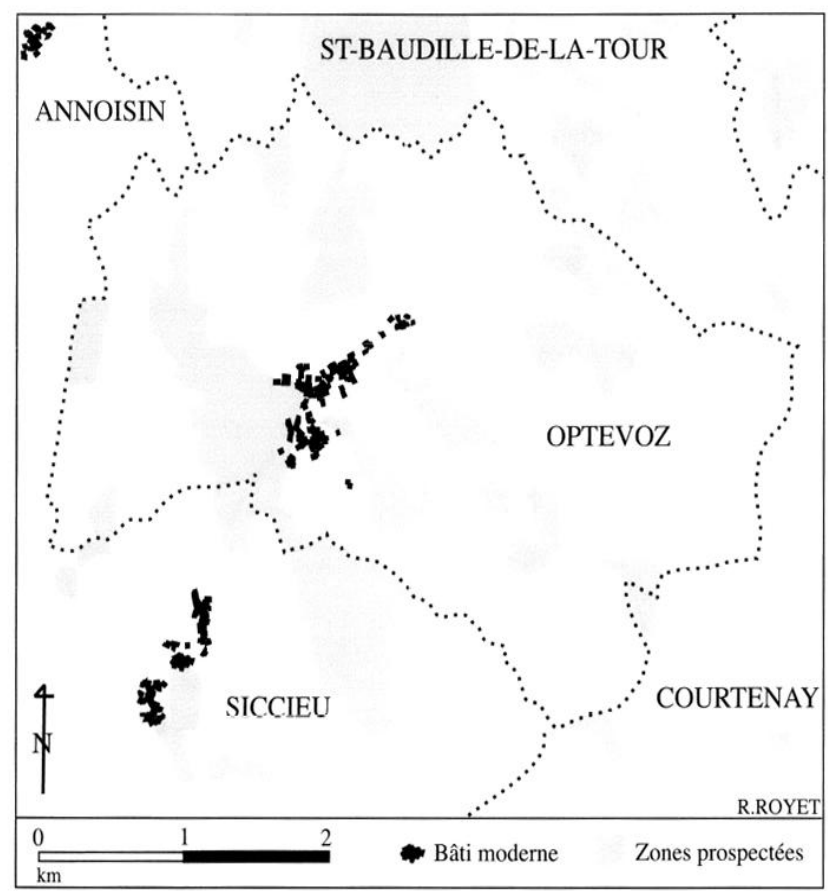

Fig. 3. Carte des zones prospectées.

reste de l'Isle Crémieu, de la part des utilisateurs de détecteurs de métaux. Le corpus de 398 monnaies et les nombrcux objets métalliques intégrés dans un récent travail universitaire (Carrara 2000) permettent de mesurer l'ampleur du pillage en cours.

\section{LE CONTEXTE ARCHÉOLOGIQUE}

\subsection{Optevoz dans la cité de Vienne}

Les récents travaux (Rémy 2000) sur les élites allobroges confirment que le Haut-Rhône marque, vraisemblablement en rive droite à quelques enclaves près, la limite du territoire allobroge puis celle de la cité de Vienne. La rive gauche du fleuve et l'Isle Crémieu qui la surplombe, tout en étant relativement éloignées du centre politique, sont toutefois un couloir de voies de communication assez densément occupé.

Pour la période laténienne, la connaissance des axes de circulation reste schématique (fig. 2). La seule voie attestée par l'archéologie borde la rive gauche du fleuve qu'elle remonte au moins jusqu'à Lagnieu. Il est difficile d'assurer que les nombreux gués attestés à l'époque moderne furent tous des points de passage fréquentés dès la protohistoire (Royet, Faucher 2002). Seul le gué de SaintOyand, autour duquel s'étend une importante nécropole tumulaire (Perrin, Schöenfelder 2003), est indubitablement utilisé depuis l'âge du Bronze pour la traversée du fleuve.

La position de l'oppidum de Larina, situé sur un éperon dominant le Rhône au débouché du val d'Amby (fig. 2), ainsi que la découverte ancienne d'une tombe gauloise en un point inconnu de la commune d'Optevoz (Müller 1931), ont conduit certains auteurs à proposer l'hypothèse d'un axe traversant le plateau (Porte 1981). Franchissant le Rhône au niveau de Saint-Vulbas, l'itinéraire en provenance de Franche-Comté remonterait par le Val d'Amby pour rejoindre Aoste et, au delà, les cols alpins. En fait, si l'existence d'un chemin empruntant le Val d'Amby ne paraît pas devoir être remise en question, il est certainement nécessaire d'en réevaluer l'utilité. À l'exception notable de l'oppidum de Larina, la plaine a livré largement autant de découvertes isolées et de sites laténiens que le plateau. Il n'est pas possible, sur un document à échelle moyenne, de restituer une concentration qui révèlerait un axe de pénétration à travers l'IsleCrémieu au vu de ces trouvailles.

Le contexte est mieux documenté pour l'époque galloromaine même si aucune route n'est signalée dans les itinéraires antiques et bien que ce tronçon de la vallée soit ignoré dans l'historiographie traditionnelle (Buisson, Pelletier 1983). Relevant administrativement de la cité de Vienne, ce secteur est aussi dans la sphère d'influence de la colonie de Lyon. Des épitaphes de notables lyonnais et de membres de leur familias, retrouvées sur la rive gauche (décurion lyonnais à La-Balme-les-Grottes, $c f$. ILN, Vienne 555), ou celles de viennois découvertes dans l'Ain (décurion viennois à Saint-Vulbas, $c f$. CIL XIII 2453), attestent de la porosité des limites entre cités. Cette double influence est lisible dans le réseau routier qui encadre l'Isle-Crémieu (fig. 2). L'ancienne piste protohistorique longeant le Rhône sur la rive gauche est reprise après la conquête. Elle peut être suivie jusqu'à un coude du fleuve à Vertrieu. Sur l'actuelle commune de Saint-Romain-deJalionas, un important nœud routier se constitue à proximité du fleuve. Deux voies nouvelles, provenant l'une de Lyon (actuelle D517) et l'autre de Vienne (qui deviendra au Moyen Âge la voie de pèlerinage de Saint-Oyand), convergent vers le gué de Saint-Oyand pour s'y rejoindre. Un troisième axe, provenant de Lyon et bordant le Rhône en aval, rejoint les précédents un peu avant leur convergence. À partir de ce croisement, une route contourne le massif de l'Isle-Crémieu par le sud pour, peut être, relier directement Lyon et Aoste (Michel 1987). Dans le secteur étudié, cette voie paraît reprise, grosso modo, par l'actuelle D517.

L'Isle-Crémieu apparaît ainsi comme un obstacle naturel de taille réduite assez facilement contournable. Les chemins empruntant les vallées (notamment les vals d'Amby ou d'Amblérieu) pour assurer une communication entre plaine et sommet du plateau paraissent donc s'inscrire dans une logique de desserte locale plutôt que de commerce à longue distance. Au contraire de la rive droite où les vicus de Briord, Lagnieu, Saint-Vulbas quadrillent assez régulièrement l'espace, la rive allobroge apparaît dépourvue d'agglomération entre Aoste et Lyon. 


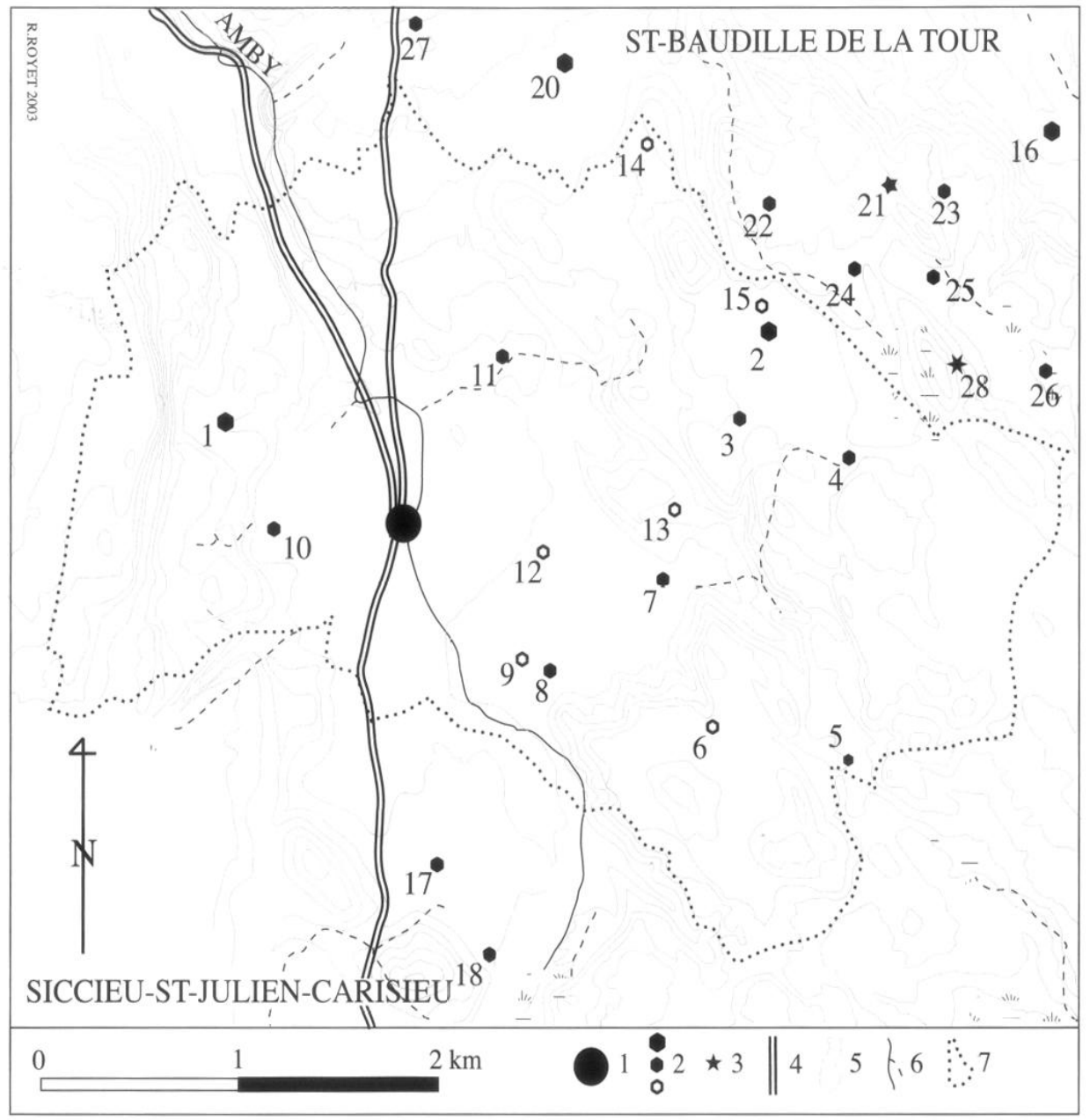

Fig. 4. Carte archéologique du bassin d'Optevoz. 1, agglomération antique ; 2 , villae-fermes-dépendances; 3 , sanctuaire?; 4 , voies; 5 , courbes de niveaux (pas de $10 \mathrm{~m}) ; 6$, rivières/rus; 7 , limite de commune.
Un réexamen récent du seul vicus supposé, situé sous le hameau de Travers à La-Balme-les-Grottes, a conduit à remettre en question son existence (Royet, Faucher 2002).

Le village moderne d'Optevoz s'étire le long d'une voie ancienne, intitulée "Chemin des Romains" sur la carte au $1 / 25000^{\mathrm{e}}$ de l'IGN. L'ancienneté de cette route est certaine même si l'appellation en est apocryphe. Cette voie qui ne subsiste parfois que sous forme d'une haie, dessine une ligne orientée grossièrement Nord-Sud desservant la partie occidentale du plateau. Reconnue sur environ onze kilomètres entre le village de Saint-Baudillede-la-Tour au nord et le hameau de Cozance (commune de Trept), elle semble se brancher sur la voie de Lyon à Aoste (fig. 2) en contrebas du plateau.

La voie remontant depuis la plaine du Rhône par le Val d'Amby aboutit sur ce "Chemin des Romains" au niveau d'Optevoz. Des sondages ont recoupé un tronçon antique prolongeant la voie du Val d'Amby sur une longueur d'environ $25 \mathrm{~m}$ juste au sud de ce carrefour (chanticr $\mathrm{n}^{\circ} 1, c f$. fig. 5).

Au moins deux autres axes suggérés par la topographie mais dont la réalité antique est toutefois hypothétique ont été proposés (Carrara 2000). Le premier, préfigurant peu ou prou l'actuel D52, desservirait Optevoz par le sud en passant par le vallon de Ry jusqu'à Crémieu. Le second, correspondant au D140b, partirait depuis Optevoz en direction du sud-est vers Courtenay. Cette seconde proposition est affaiblie par l'absence de vestiges, fossé ou chaussée, dans les sondages effectués de part et d'autre de la route actuelle (chantiers $n^{\circ} 5$ et $8, c f$. fig. 5).

Signalons enfin qu'aucun système parcellaire orthonormé n'a été identifié sur le plateau.

\subsection{Carte archéologique du bassin d'Optevoz}

À ce jour les prospections ont permis d'examiner la majeure partie des terres cultivées du secteur étudié, soit environ 350 ha (fig. 3). Les lacunes importantes constatées à l'est et à l'ouest correspondent essentiellement à des zones à fort pendage: moraines, bourrelet en pied de falaise, affleurement calcaire respectivement couverts de taillis, de prés ou de pelouses sèches peu propices à la détection.

Malgré la faible proportion du territoire communal examiné, la carte archéologique disponible semble représentative de la réalité antique. Les sommets et les pentes de buttes ou encore les secteurs au rocher affleurant apparaissent en effet peu propices à des habitats. 

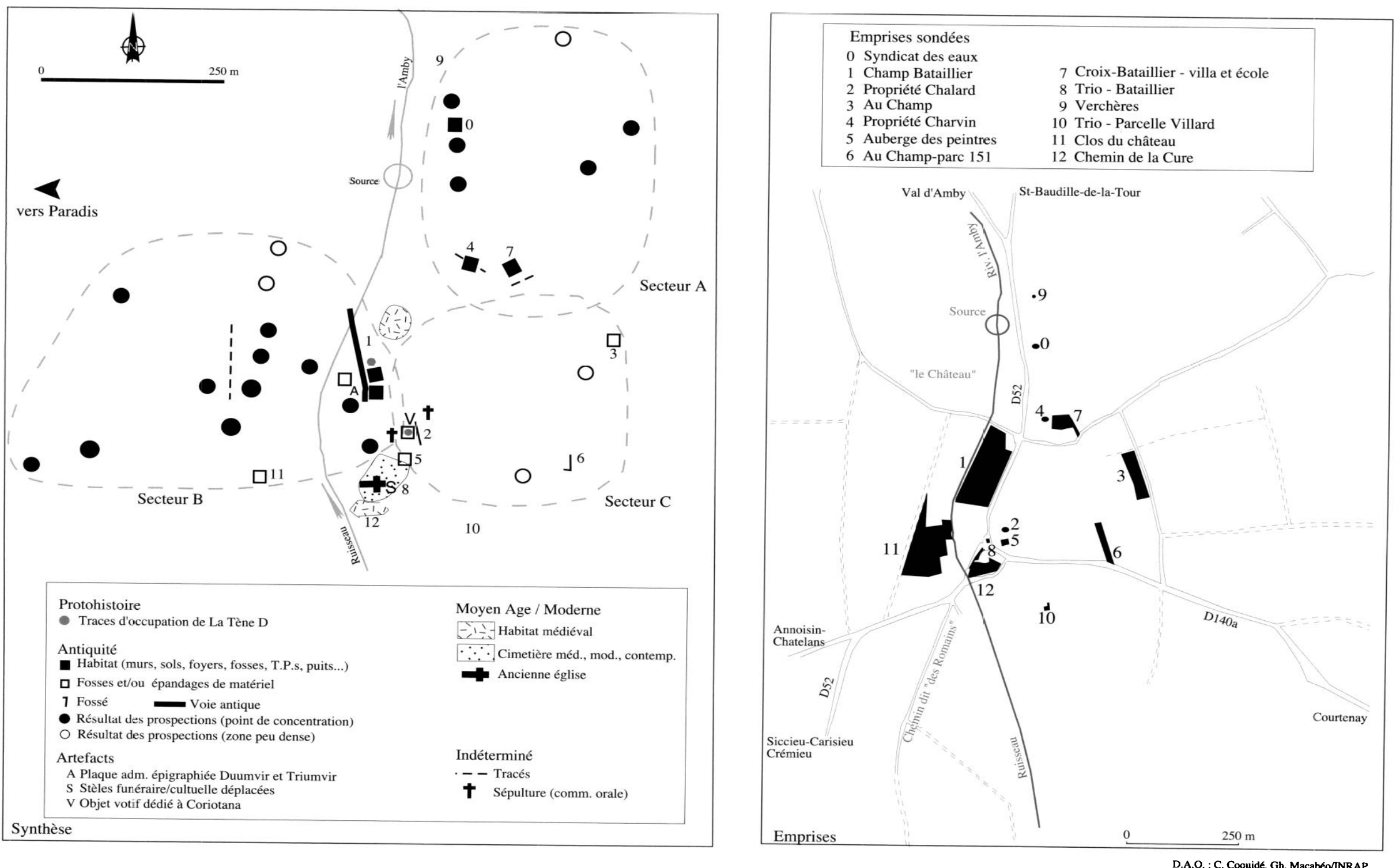

Fig. 5. Optevoz. Synthèse archéologique et emprise des chantiers. 
Les tableaux suivants ${ }^{2}$ récapitulent les principales caractéristiques des sites identifiés (fig. 4). Il comporte, en plus des gisements situés dans la doline d'Optevoz,

plusieurs sites repérés hors de cette zone mais importants pour en comprendre l'organisation.

\section{Commune d'Optevoz}

\begin{tabular}{|c|c|c|c|c|c|c|}
\hline Lieu-dit et parcelle & C. L. & Surface & $\begin{array}{c}\text { Indices } \\
\text { architecturaux }\end{array}$ & Matériel & Datation & $\begin{array}{l}\text { Catégorie } \\
\text { de site }\end{array}$ \\
\hline $\begin{array}{l}\text { Le Paradis } \\
\text { A107 à } 112,119\end{array}$ & $\begin{array}{l}X: 831,950 \\
Y: 2088,500\end{array}$ & $1,5 \mathrm{ha}$ & $\begin{array}{l}\text { tegulae, pierres, } \\
\text { enduits peints, tubuli, } \\
\text { tuileau, marbre }\end{array}$ & $\begin{array}{l}\text { céramique, verre, } \\
\text { dolia, scories, } \\
\text { meules, monnaies } \\
\text { (trésor), bijoux, } \\
\text { vaisselle métallique }\end{array}$ & $\begin{array}{l}\mathrm{I}^{\mathrm{er}}-\mathrm{III}^{\mathrm{e}} \mathrm{s} \text {. } \\
\text { réoccupation Haut } \\
\text { Moyen Âge }\end{array}$ & $\begin{array}{l}\text { villa première } \\
\text { catégorie }\end{array}$ \\
\hline $\begin{array}{l}\text { Lécherolles, C160 à } \\
167,174,179 \text { à } 182\end{array}$ & $\begin{array}{l}X: 834,530 \\
Y: 2088,960\end{array}$ & 0,5 ha & $\begin{array}{l}\text { tegulae, pierres, } \\
\text { tubuli, tuileau, tuf }\end{array}$ & $\begin{array}{l}\text { céramique, dolia, } \\
\text { scories, meules }\end{array}$ & $\mathrm{I}^{\mathrm{er}} \mathrm{s} . \mathrm{av} .-\mathrm{IV}^{\mathrm{e}} \mathrm{s}$. & $\begin{array}{l}\text { villa deuxième } \\
\text { catégorie }\end{array}$ \\
\hline $\begin{array}{l}\text { Sur Sollier } \\
\text { D335, } 336\end{array}$ & $\begin{array}{l}X: 834,600 \\
Y: 2088,500\end{array}$ & 0,3 ha & tegulae, pierres & céramique & $\mathrm{I}^{\mathrm{er}}-\mathrm{II}^{\mathrm{e}} \mathrm{S}$ & ferme \\
\hline $\begin{array}{l}\text { Catelinière } \\
\text { C } 374,375,379 \text {, } \\
380,382,383\end{array}$ & $\begin{array}{l}X: 835,00 \\
Y: 2088,300\end{array}$ & $1000 \mathrm{~m}^{2}$ & tegulae & $\begin{array}{l}\text { céramique, scories, } \\
\text { sonnailles }\end{array}$ & $\mathrm{I}^{\mathrm{er}}-\mathrm{II}^{\mathrm{e}} \mathrm{s}$. & ferme \\
\hline $\begin{array}{l}\text { La Foulure } \\
\text { E102 }\end{array}$ & $\begin{array}{l}X: 835,25 \\
Y: 2086,900\end{array}$ & $300 \mathrm{~m}^{2}$ & tegulae & & & $\begin{array}{l}\text { dépendance } \\
\text { agricole }\end{array}$ \\
\hline $\begin{array}{l}\text { Côtes Reynaud } \\
\text { E611, } 620\end{array}$ & $\begin{array}{l}X: 835,550 \\
Y: 2087,00\end{array}$ & $700 \mathrm{~m}^{2}$ & tegulae & céramique & & $\begin{array}{l}\text { dépendance } \\
\text { agricole }\end{array}$ \\
\hline $\begin{array}{l}\text { La Fouesse } \\
\text { E1 et } 2\end{array}$ & $\begin{array}{l}X: 834,150 \\
Y: 2087,750\end{array}$ & $1500 \mathrm{~m}^{2}$ & tegulae, pierres & $\begin{array}{l}\text { céramique, verre, } \\
\text { scories, meules, } \\
\text { monnaies }\end{array}$ & $\begin{array}{l}\mathrm{I}^{\mathrm{er}} \mathrm{s} . \text { av. - I } \mathrm{I}^{\mathrm{er}} \mathrm{s} \text {. ap. } \\
\text { réoccupation } \mathrm{IV}^{\mathrm{e}} \mathrm{s} .\end{array}$ & ferme \\
\hline $\begin{array}{l}\text { Le Couloup, Bizieu } \\
\text { E333 à } 337\end{array}$ & $\begin{array}{l}X: 833,725 \\
Y: 2087,225\end{array}$ & $1500 \mathrm{~m}^{2}$ & tegulae, pierres & $\begin{array}{l}\text { céramique, verre, } \\
\text { dolia, scories, } \\
\text { meules, monnaies }\end{array}$ & $\mathrm{I}^{\mathrm{er}} \mathrm{s}$. av. $-\mathrm{IV}^{\mathrm{e}} \mathrm{s}$. & ferme \\
\hline $\begin{array}{l}\text { Bizieu } \\
\text { E363 à } 372\end{array}$ & $\begin{array}{l}X: 833,575 \\
Y: 2087,250\end{array}$ & $3000 \mathrm{~m}^{2}$ & tegulae & & & dépendance \\
\hline $\begin{array}{l}\text { Les Rochettes } \\
\text { E265 }\end{array}$ & $\begin{array}{l}X: 832,125 \\
Y: 2087,825\end{array}$ & $2500 \mathrm{~m}^{2}$ & tegulae & $\begin{array}{l}\text { céramique, scories, } \\
\text { meules }\end{array}$ & $\mathrm{I}^{\mathrm{er}}-\mathrm{II}^{\mathrm{e}} \mathrm{s}$ & ferme \\
\hline $\begin{array}{l}\text { Pré du Clos } \\
\text { C286, } 287\end{array}$ & $\begin{array}{l}X: 833,175 \\
Y: 2089,200\end{array}$ & $3000 \mathrm{~m}^{2}$ & tegulae, pierres & $\begin{array}{l}\text { céramique, scories, } \\
\text { meules, bijoux }\end{array}$ & $\mathrm{I}^{\mathrm{er}}-\mathrm{III}^{\mathrm{e}} \mathrm{s}$. & ferme \\
\hline $\begin{array}{l}\text { Boursent } \\
\text { E457 à } 459\end{array}$ & $\begin{array}{l}X: 833,600 \\
Y: 2087,800\end{array}$ & $500 \mathrm{~m}^{2}$ & tegulae, pierres & & & dépendance \\
\hline $\begin{array}{l}\text { Ferrouillet } \\
\text { D517, 518 }\end{array}$ & $\begin{array}{l}X: 834,225 \\
Y: 2088,00\end{array}$ & $100 \mathrm{~m}^{2}$ & tegulae & & & dépendance \\
\hline $\begin{array}{l}\text { Bonnet du Loup } \\
\text { C18 }\end{array}$ & $\begin{array}{l}X: 834,075 \\
Y: 2089,800\end{array}$ & $100 \mathrm{~m}^{2}$ & tegulae & & & dépendance \\
\hline $\begin{array}{l}\text { Tillieux } \\
\text { C109 }\end{array}$ & $\begin{array}{l}X: 834,500 \\
Y: 2089,125\end{array}$ & $100 \mathrm{~m}^{2}$ & tegulae & & & dépendance \\
\hline
\end{tabular}

\section{Commune de Courtenay}

\begin{tabular}{|c|c|c|c|c|c|c|}
\hline Lieu-dit et parcelle & C. L. & Surface & $\begin{array}{c}\text { Indices } \\
\text { architecturaux }\end{array}$ & Matériel & Datation & $\begin{array}{l}\text { Catégorie } \\
\text { de site }\end{array}$ \\
\hline Les Prés de Planchue & $\begin{array}{l}X: 836,150 \\
Y: 2089,950\end{array}$ & 4 à 5 ha & $\begin{array}{l}\text { tegulae, pierres, } \\
\text { enduits peints, tubuli, } \\
\text { tuileau, marbre, } \\
\text { mosaïque }\end{array}$ & $\begin{array}{l}\text { céramique, verre, } \\
\text { dolia, scories, } \\
\text { meules, monnaies, } \\
\text { bijoux, vaisselle } \\
\text { métallique, outils }\end{array}$ & $\mathrm{I}^{\mathrm{er}}-\mathrm{IV}^{\mathrm{e}} \mathrm{s}$ & $\begin{array}{l}\text { villa première } \\
\text { catégorie }\end{array}$ \\
\hline
\end{tabular}

2 Tableaux réalisés notamment d’après les travaux inédits de R. Convert (fiches manuscrites conservées au SRA Rhône-Alpes) pour les sites 2 à 6 , 9 à $15,17,18,20,24,25,27$ et après le réexamen du corpus par $\mathrm{S}$. Carrara qui a signalé les sites 7,8, 16, 21 à 23, 26 (Carrara 2000). 
Commune de Siccieu-Saint-Julien-Carizieu

\begin{tabular}{|c|c|c|c|c|c|c|}
\hline Lieu-dit et parcelle & C. L. & Surface & $\begin{array}{c}\text { Indices } \\
\text { architecturaux }\end{array}$ & Matériel & Datation & $\begin{array}{c}\text { Catégorie } \\
\text { de site }\end{array}$ \\
\hline $\begin{array}{l}\text { Champagne 1, A172 } \\
\text { à } 177,180-181\end{array}$ & $\begin{array}{l}X: 833,150 \\
Y: 2086,250\end{array}$ & $7000 \mathrm{~m}^{2}$ & tegulae, pierres & céramique, monnaies & $\begin{array}{l}I^{\text {er }} s . \text { av. }-{ }^{\text {er }} s . \text { ap. } \\
\text { réoccupation } I^{\mathrm{e}} s \text {. }\end{array}$ & ferme \\
\hline $\begin{array}{l}\text { Champagne 2, A230 } \\
\text { à } 235,256,258\end{array}$ & $\begin{array}{l}X: 833,375 \\
Y: 2085,800\end{array}$ & $1800 \mathrm{~m}^{2}$ & tegulae & $\begin{array}{l}\text { céramique, } \\
\text { poids en bronze }\end{array}$ & & ferme \\
\hline $\begin{array}{l}\text { Gillieu } \\
\text { B8 à } 10\end{array}$ & $\begin{array}{l}X: 830,650 \\
Y: 2086,950\end{array}$ & $2000 \mathrm{~m}^{2}$ & tombes & céramique & $\mathrm{III}^{\mathrm{e}}-\mathrm{IV}^{\mathrm{e}} \mathrm{s}$ & nécropole \\
\hline
\end{tabular}

Commune de Saint-Baudille-de-la-Tour

\begin{tabular}{|c|c|c|c|c|c|c|}
\hline Lieu-dit et parcelle & C. L. & Surface & $\begin{array}{c}\text { Indices } \\
\text { architecturaux }\end{array}$ & Matériel & Datation & $\begin{array}{l}\text { Catégorie } \\
\text { de site }\end{array}$ \\
\hline $\begin{array}{l}\text { Charnioux-La Plaine } \\
\text { E200 à 203, } 253 \text { à } \\
267,400 \text { à } 402\end{array}$ & $\begin{array}{l}X: 833,580 \\
Y: 2090,190\end{array}$ & 4,5 ha & $\begin{array}{l}\text { tegulae, pierres, } \\
\text { mosaïque, enduits } \\
\text { peints, tubuli, tuileau }\end{array}$ & $\begin{array}{l}\text { céramique, verre, } \\
\text { scories, meules, } \\
\text { monnaies, bijoux }\end{array}$ & $\mathrm{I}^{\mathrm{er}} \mathrm{s} . \mathrm{av} .-\mathrm{V}^{\mathrm{e}} \mathrm{s}$. & $\begin{array}{l}\text { villa première } \\
\text { catégorie }\end{array}$ \\
\hline $\begin{array}{l}\text { Marcolay } \\
\text { D191, } 198\end{array}$ & $\begin{array}{l}X: 835,575 \\
Y: 2089,580\end{array}$ & $200 \mathrm{~m}^{2}$ & tegulae, pierres & $\begin{array}{l}\text { céramique, } \\
75 \text { monnaies }\end{array}$ & $\mathrm{I}^{\mathrm{er}} \mathrm{s} . \mathrm{av} .-\mathrm{II}^{\mathrm{e}}-\mathrm{IV}^{\mathrm{e}} \mathrm{s}$. & sanctuaire \\
\hline $\begin{array}{l}\text { Noyaret } \\
\text { D122, } 123\end{array}$ & $\begin{array}{l}X: 835,850 \\
Y: 2089,300\end{array}$ & $2000 \mathrm{~m}^{2}$ & tegulae & céramique & $\mathrm{I}^{\mathrm{er}}-\mathrm{IV}^{\mathrm{e}} \mathrm{s}$ & ferme \\
\hline $\begin{array}{l}\text { Chevrier } \\
\text { E374, 385, } 386\end{array}$ & $\begin{array}{l}X: 835,850 \\
Y: 2089,300\end{array}$ & $7500 \mathrm{~m}^{2}$ & tegulae & céramique, monnaies & $\mathrm{I}^{\mathrm{er}}-\mathrm{II}^{\mathrm{e}}-\mathrm{IV}^{\mathrm{e}} \mathrm{s}$ & ferme \\
\hline $\begin{array}{l}\text { Lacliou } \\
\text { D182 }\end{array}$ & $\begin{array}{l}X: 835,500 \\
Y: 2089,175\end{array}$ & $2000 \mathrm{~m}^{2}$ & tegulae & $\begin{array}{l}\text { céramique, scories, } \\
\text { meules, monnaies }\end{array}$ & $\mathrm{I}^{\mathrm{er}}-\mathrm{IV}^{\mathrm{e}} \mathrm{s}$ & $\begin{array}{l}\text { ferme ou atelier } \\
\text { métallurgique }\end{array}$ \\
\hline $\begin{array}{l}\text { Levarey } \\
\text { D173, } 174\end{array}$ & $\begin{array}{l}X: 836,175 \\
Y: 2088,675\end{array}$ & $7500 \mathrm{~m}^{2}$ & tegulae, pierres & $\begin{array}{l}\text { céramique, verre, } \\
\text { scories, meules, } \\
\text { monnaies }\end{array}$ & $\mathrm{I}^{\mathrm{er}}-\mathrm{IV}^{\mathrm{e}} \mathrm{s}$ & $\begin{array}{l}\text { ferme ou atelier } \\
\text { métallurgique }\end{array}$ \\
\hline $\begin{array}{l}\text { Les Mézières } \\
\text { E103 }\end{array}$ & $\begin{array}{l}X: 832,800 \\
Y: 2090,275\end{array}$ & $2000 \mathrm{~m}^{2}$ & tegulae & $\begin{array}{l}\text { céramique, meules, } \\
\text { monnaies }\end{array}$ & $\mathrm{I}^{\mathrm{er}} \mathrm{s} . \mathrm{av} .-\mathrm{IV}^{\mathrm{e}} \mathrm{s}$. & ferme \\
\hline Marcolay 2 & $\begin{array}{l}X: 835,375 \\
Y: 2089,580\end{array}$ & & tombes en lauzes & bijoux & $\mathrm{IV}^{\mathrm{e}} \mathrm{s}$ & cimetière \\
\hline
\end{tabular}

\section{L'AGGLOMÉRATION ANTIQUE}

Il n'existe que très peu de documentation ancienne sur le village proprement dit. Les découvertes réalisées au cours de travaux n'ont été rapportées que de façon très laconique et imprécise. Dans plusieurs cas on ne dispose que d'informations transmises oralement et sujettes à caution. Aucun vestige immobilier ancien n'est actuellement visible. Seuls deux éléments funéraires dont la provenance originelle reste inconnue sont conservés à l'intérieur de l'église. L'un est une stèle funéraire (ILN, Vienne 567) jadis réemployée comme piédroit d'une baie jumelle du clocher de l'église romane et déposée lors de la reconstruction de l'édifice ${ }^{3}$. Le second est une stèle anépigraphe (autel ou plus vraisemblablement cippe), réputée avoir été récupérée lors des mêmes travaux ${ }^{4}$.

L'essenticl des données disponibles sur le site antique provient donc des évaluations d'archéologie préventive.
Une zone de protection dessinant un quadrilatère d'environ $800 \mathrm{~m}$ de côté, couvre la totalité du village actuel et les terrains avoisinants. Le vide archéologique de 500 à $700 \mathrm{~m}$ constaté autour du village permet d'en fixer les limites. Depuis 1997, près de $6 \%$ de la surface protégée ont ainsi été sondés à l'occasion d'aménagements de dimensions très variables allant de la construction de piscine au lotissement ou à la réfection des espaces publics. Les solutions techniques de conservation ayant été privilégiées, aucune fouille n'a été mise en place à la suite de ces évaluations. En complément de ces sondages, des prospections pédestres ont fourni quelques informations sur les marges de l'agglomération.

Dans l'état actuel de la recherche, l'essentiel des données est regroupé en deux secteurs distincts (fig. 5). Le premier occupe le côté est de la D 52, au nord du village, sur un rayon de $150 \mathrm{~m}$ (secteur A). Le second, formant un rectangle de $300 \mathrm{~m} \mathrm{X} 600 \mathrm{~m}$, se situe à $170 \mathrm{~m}$ vers le sud-

3 Photo ancienne non datée et anonyme de l'ancienne église détruite avant 1892.

4 Tradition orale. 


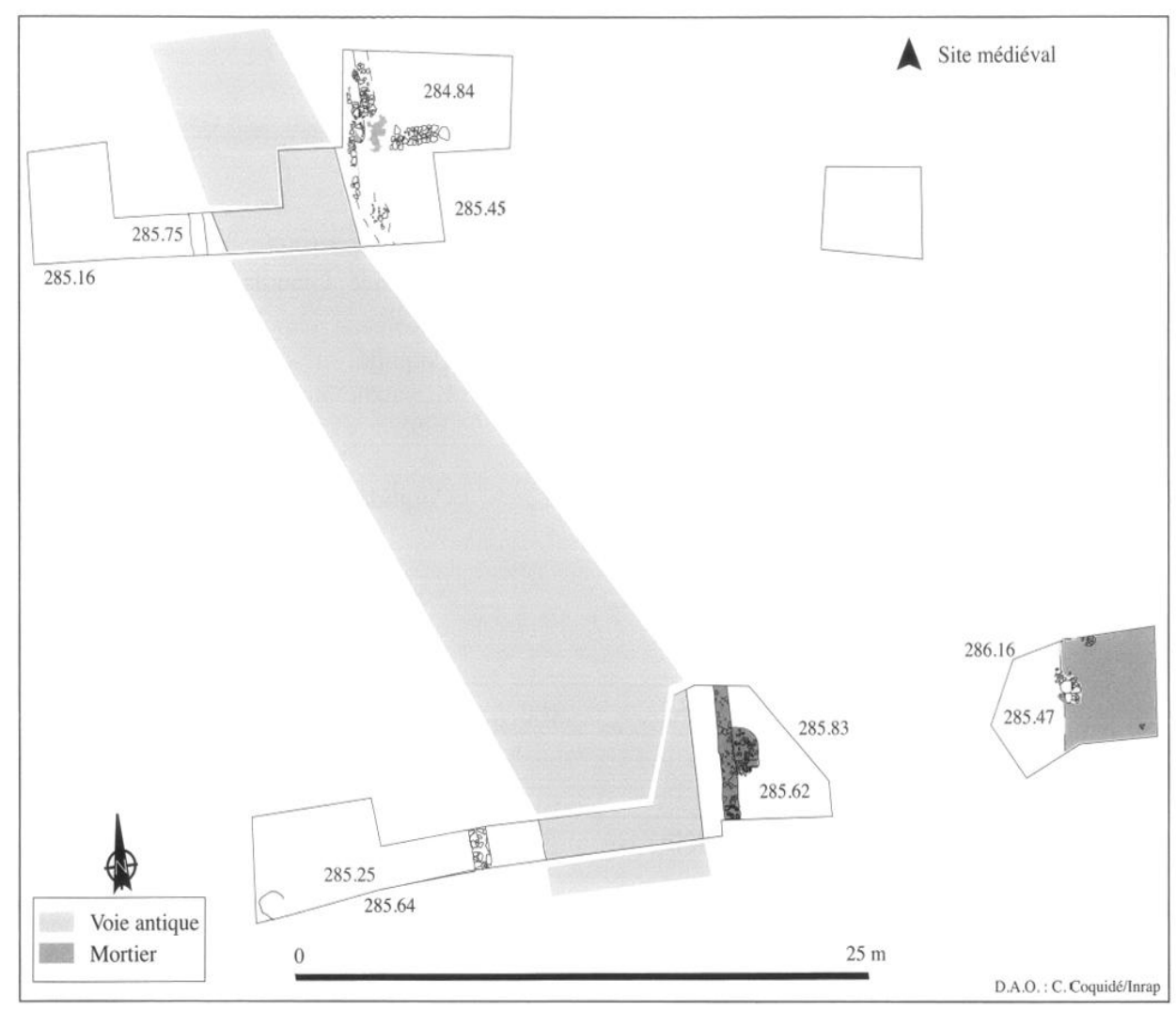

Fig. 6. Optevoz "Champ Batailler". Plan masse de l'occupation antique (chantier 1).

ouest (secteur B). Un troisième secteur (secteur C) regroupe des données plus disparates et diffuses recueillies au sud et à l'est des deux précédents.

Dans les secteurs A et B, on enregistre un habitat évoluant du Ier s. av. J.-C. au III ${ }^{\mathrm{e}}$ s. ap. J.-C. Outre de nombreux fragments céramiques et de verre on relève des témoignages d'activités domestiques comme la mouture (fragments de meule en basalte) et le tissage (poids de métier à tisser) mais aussi des traces de métallurgie (scories de fer dont plusieurs fonds de four). Il convient également de noter que des dizaines de monnaies y ont été récupérées lors de ramassages au détecteur à métaux (corpus dans Carrara 2000).

\subsection{Opérations d'évaluation archéologique sur l'agglomération antique}

On se reportera au tableau récapitulatif page suivante.

\subsection{Le secteur $A$}

Le secteur A (fig. 5) semble s'aligner sur la voirie actuelle (Chemin des Romains, $c f$. supra) dont l'origine est réputée antique. Cette impression est peut-être toutefois simplement liée à la carte de répartition des aménagements actuels.

Il a été possible d'aborder par sondage des parcelles non prospectées situées dans les jardins à l'arrière des fermes et maisons sur rue. Un habitat n'obéissant pas à une orientation unique a été mis en évidence. S'il n'est pas possible d'en restituer l'organisation, on peut toutefois en souligner certaines caractéristiques. Le chantier 7 (fig. 7) a révélé plusieurs bâtiments. Les murs, bâtis avec des moellons de calcaire bathoniens liés ou non au mortier, présentent des modes d'assemblage variés et obéissent à deux orientations légèrement divergentes. L'un d'entre eux, suivi sur près de $30 \mathrm{~m}$, suggère, à un moment donné, la structuration selon une orientation à $18^{\circ} \mathrm{W}$ des habitats ou d'un groupe d'installations à vocation particulière (artisanale ?). Un tambour de colonne réemployé pourrait d'ailleurs provenir d'un portique. Les sondages ont confirmé la présence d'un habitat dense et stratifié sur 30 à $40 \mathrm{~cm}$, bordé au nord et à l'est d'espaces extérieurs aménagés et stabilisés à l'aide de remblais limono-graveleux où fut creusé un puits.

À l'intérieur des bâtiments, les sols sont stabilisés par une succession de plusieurs sols de cailloutis et de planchers suggérés par des semis de clous. De nombreux foyers aménagés avec des soles de terre cuite ou de plaques calcaires ont été rencontrés dans une surface très réduite. L'association de ces foyers avec des fosses et divers petits fragments de métaux ferreux et cuivreux ainsi qu'une aiguille en os à trois chas suggère plutôt des activités artisanales dont la nature reste toutefois à préciser. Enfin, un aménagement composé d'un assemblage soigné de tegulae fragmentées et dessinant un quadrilatère réservant un espace central de $1,18 \mathrm{~m}$ de côtés reste non identifié à proximité (fig. 7). 


\begin{tabular}{|c|c|c|c|c|c|c|c|}
\hline $\begin{array}{c}\text { Chantier (fig. 5) } \\
\text { OPTEVOZ }\end{array}$ & $\begin{array}{l}\text { Secteur } \\
\text { antique }\end{array}$ & Résultat & Motif & $\begin{array}{l}\text { Surface } \\
\text { disp. en } \mathrm{m}^{2}\end{array}$ & \% étudié & Références & Notes \\
\hline $\begin{array}{l}\text { Syndicat des } \\
\text { eaux (chantier 0) }\end{array}$ & A & $\begin{array}{l}\text { bâti antique dense avec un } \\
\text { nombreux mobilier céra- } \\
\text { mique, métallique, verrerie... }\end{array}$ & $\begin{array}{l}\text { Syndicat } \\
\text { des eaux }\end{array}$ & & & Carrara 2000 & $\begin{array}{l}\text { destruction sans } \\
\text { surveillance }\end{array}$ \\
\hline $\begin{array}{l}\text { Champ Bataillier } \\
\text { (chantier 1) }\end{array}$ & B & $\begin{array}{l}\text { voie et bâti antique, sols, } \\
\text { puits ou silo (sud), site } \\
\text { médiéval, fosses et poteaux } \\
\left(\mathrm{X}^{\mathrm{e}}-\mathrm{XIII} \mathrm{I}^{\mathrm{e}} \mathrm{s}\right) \text { (nord) }\end{array}$ & villas & 10200 & 20 & \begin{tabular}{|l|} 
Coquidé, \\
Brouillaud 1997 \\
(Coquidé, Gianola \\
1999)
\end{tabular} & $\begin{array}{l}\text { sud détruit par } \\
\text { viabilisation } \\
\text { sauvage }\end{array}$ \\
\hline $\begin{array}{l}\text { Propriété Chalard } \\
\text { (chantier 2) }\end{array}$ & $\mathrm{B} / \mathrm{C}$ & $\begin{array}{l}\text { fosses, fossé/palissade } \\
\text { antiques ; colluvion } \\
\text { anthropisée antique }\end{array}$ & piscine & 78 & 100 & $\begin{array}{l}\text { Coquidé, } \\
\text { Kovatchévitch } \\
1998 \\
\end{array}$ & \\
\hline $\begin{array}{l}\text { Au Champ } \\
\text { (chantier 3) }\end{array}$ & $\mathrm{C}$ & $\begin{array}{l}\text { fosses antiques peu pro- } \\
\text { fondes, rares céramiques }\end{array}$ & villas & 4000 & 12 & Coquidé 1998 & \\
\hline $\begin{array}{l}\text { Propriété Charvin } \\
\text { (chantier 4) }\end{array}$ & A & $\begin{array}{l}\text { voie ou sol (?) } \\
\text { et bâti antique }\end{array}$ & piscine & strati. & & Nourissat 1999 & $\begin{array}{l}\text { relevés après } \\
\text { travaux }\end{array}$ \\
\hline $\begin{array}{l}\text { Auberge des } \\
\text { Peintres } \\
\text { (chantier 5) }\end{array}$ & $\mathrm{B} / \mathrm{C}$ & $\begin{array}{l}\text { fosse-dépotoir d'éléments de } \\
\text { construction et sépultures } \\
\text { médiévales en coffre mixte ; } \\
\text { colluvion anthropisée antique }\end{array}$ & $\begin{array}{l}\text { aménagement } \\
\text { d'annexes } \\
\text { à l'auberge }\end{array}$ & strati. & 100 & $\begin{array}{l}\text { Coquidé, Guyon } \\
1999\end{array}$ & \\
\hline $\begin{array}{l}\text { Au Champ } \\
\text { parcelle 151 } \\
\text { (chantier 6) }\end{array}$ & $\mathrm{C}$ & $\begin{array}{l}\text { enclos et empierrement } \\
\text { antiques }\end{array}$ & villa & 1700 & 18 & $\begin{array}{l}\text { Coquidé, Faletto } \\
2000\end{array}$ & \\
\hline $\begin{array}{l}\text { Croix Bataillier } \\
\text { parc. 392-424 } \\
\text { École (chantier 7) }\end{array}$ & A & $\begin{array}{l}\text { bâti antique stratifié, } \\
\text { avec murs, sols, foyers, } \\
\text { fosses, puits, voirie }\end{array}$ & villa puis école & 2873 & 100 & \begin{tabular}{|l|} 
Coquidé, Poole \\
2002 ; Coquidé, \\
Bonnet, Joly 2004 \\
\end{tabular} & stratifié \\
\hline $\begin{array}{l}\text { Trio-Bataillier } \\
\text { (chantier 8) }\end{array}$ & & $\begin{array}{l}\text { états de l'église ant. à } 1892, \\
2 \text { cimetières du VIII' }^{\mathrm{e}} \text { au } \\
\text { XIX }^{\mathrm{e}} \text { s., fossé d'enceinte, } \\
\text { voies médiévales, modernes }\end{array}$ & $\begin{array}{l}\text { réfection de } \\
\text { la place }\end{array}$ & 3130 & 4,5 & $\begin{array}{l}\text { Coquidé, Savino } \\
2003\end{array}$ & stratifié \\
\hline $\begin{array}{l}\text { Verchères } \\
\text { (chantier 9) }\end{array}$ & Nord A & deux fragments de tegulae & piscine + annexe & 119,6 & 22,6 & Bleu 2003 & \\
\hline $\begin{array}{l}\text { Trio, parc. Villard } \\
\text { (chantier 10) }\end{array}$ & & aucun vestige ou matériel & stabulation & 842 & 15 & $\begin{array}{l}\text { Coquidé, Lalaï } \\
\text { 2003a }\end{array}$ & \\
\hline $\begin{array}{l}\text { Clos du Château } \\
\text { (chantier 11) }\end{array}$ & Sud B & $\begin{array}{l}\text { épandages de tegulae et } \\
\text { graviers triés (extraction), } \\
\text { rares trous de poteau }\end{array}$ & lotissement & 11103 & 6,6 & $\begin{array}{l}\text { Coquidé, Franc, } \\
\text { Lalaï } 2003\end{array}$ & \\
\hline $\begin{array}{l}\text { Chemin } \\
\text { de la Cure } \\
\text { (chantier 12) }\end{array}$ & & $\begin{array}{l}\text { site médiéval non daté (fosses } \\
\text { et poteaux), fossé d'enceinte } \\
\text { ecclésiale, sépultures médié- } \\
\text { vales en coffre de dalles, bâti } \\
\text { moderne/contemporain }\end{array}$ & parking & 1595 & 11,6 & $\begin{array}{l}\text { Coquidé, Lalaï } \\
\text { 2003b }\end{array}$ & \\
\hline
\end{tabular}

Prolongeant l'extension du chantier précédent, un relevé des stratigraphies des parois de la fosse d'une piscine, effectué dans l'urgence, a permis de noter un niveau de sol et une fondation de pierres calcaires et de briques non liées orientée NNE/SSW (chantier $n^{\circ} 4$ ). Plus au Nord, la construction sans surveillance archéologique d'un ensemble de bâtiments et le déplacement des déblais en décharge a livré un grand nombre de céramiques (tessons et poids de tisserands), de blocs calcaires, de tegulae, de fragments de meule et de mobilier métallique (monnaie, fibule, scories de fer) indiquant la destruction d'un autre secteur bâti (chantier $n^{\circ} 0$ ). Enfin, deux chaussées ou espaces damés ont été identifiés à l'intérieur de ce péri- mètre: un radier hétérogène antique sur le chantier $n^{\circ} 4$ (or.: NW/SE) et un épais niveau graveleux mal daté dans l'accès à la parcelle du chantier $n^{\circ} 7$ (or.: ENE/WSW).

\subsection{Le secteur B}

Le secteur B (fig. 5) se développe de part et d'autre du ruisseau des Moulins. Le tracé de ce cours d'eau, contraint par les courbes topographiques, ne peut avoir beaucoup varié au cours des derniers siècles. Les stratigraphies des chantiers 1 et 11 ont révélé des rives peu accentuées mais exhaussées d'épais limons bruns auxquels se mêlent de nombreux fragments de céramiques et de tegulae, apports 


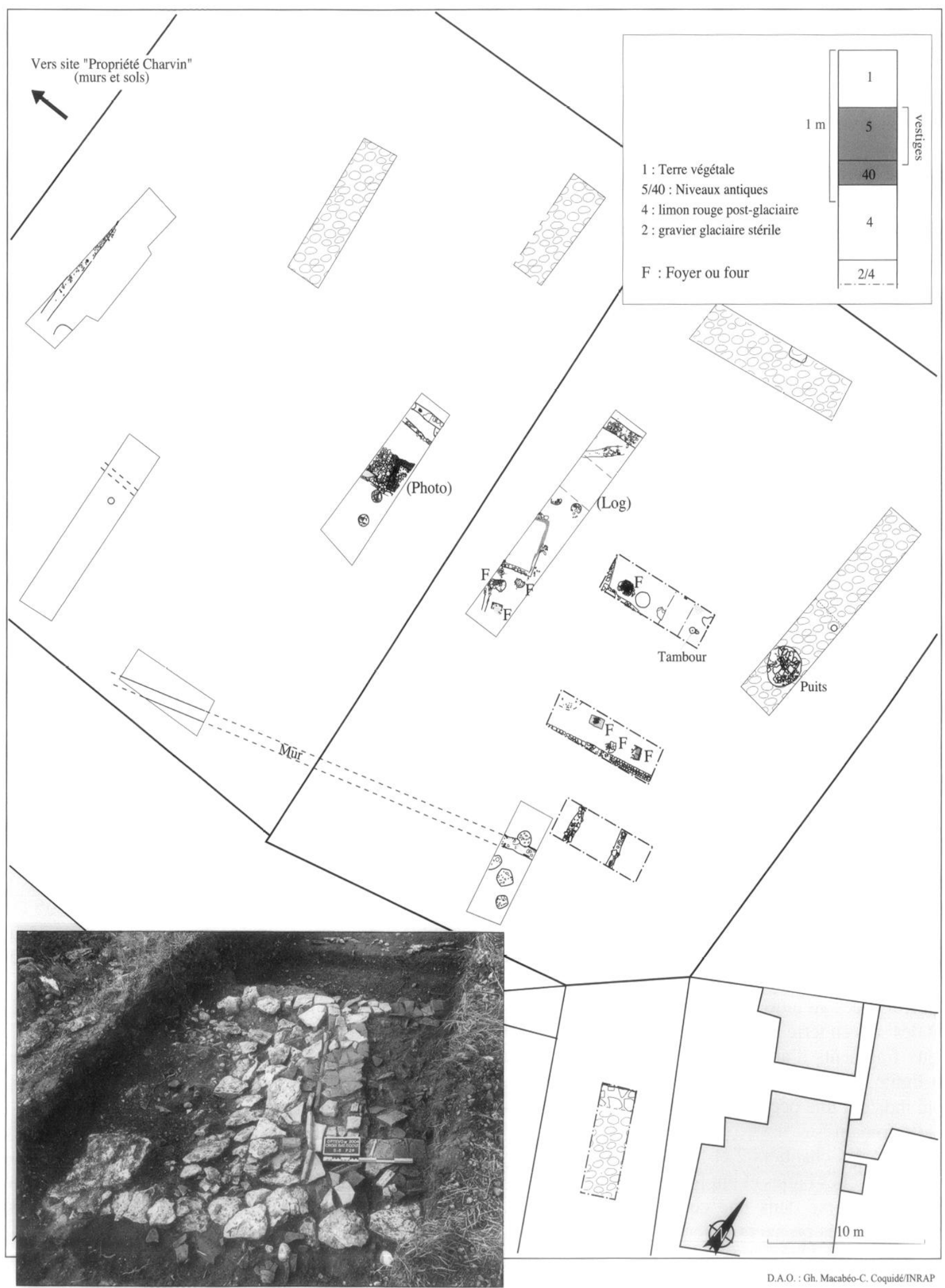

Fig. 7. Optevoz "Croix Bataillier". Plan masse (chantier 7). 


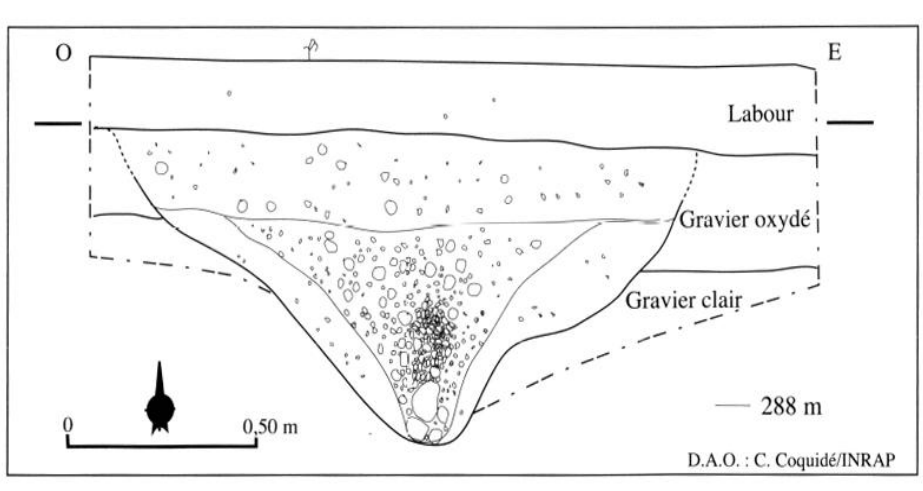

Fig. 8. Optevoz "Au Champ-Parc. 151". Enclos (chantier 6).

qui ont nivelé les surfaces et resserré le lit selon un profil proche de l'actuel.

Sur la rive gauche, des prospections pédestres signalent des poches d'occupation de part et d'autre d'un "empierrement" de dix à quinze mètres de large d'orientation globalement nord-sud. Les sondages effectués sur l'emprise du chantier 11 ne livrent toutefois que de rares trous de poteaux et fosses. Ceux-ci témoignent d'une occupation ponctuelle des bords de la rivière. Cette zone située en périphérie a essentiellement donné lieu à l'extraction des sables et graviers. Les épandages de galets ou de tegulae qui y ont été relevés ne paraissent pas pouvoir être rattachés à des aménagements ou des constructions. Ils témoignent simplement de tentatives de régularisation du sol perturbé.

Sur la rive droite, le bâti s'articule le long d'une voie (chantier $\mathrm{n}^{\circ} 1, c f$. fig. 6). Son orientation à environ $20^{\circ} \mathrm{W}$ peut conduire à voir dans ce tronçon l'extrémité de la route remontant de la plaine par le Val d'Amby. De six à huit mètres de large, sa chaussée se compose de niveaux de dallettes et paraît limitée par des murets. Cette voie est bordée par un habitat transformé à plusieurs reprises. Celui-ci se développe sur un léger relief et, bien que non stratifié, semble bien conservé par endroits. Un puits (ou un silo) en pierres sèches et des fondations en pierres calcaires parfois liées au mortier associées à quelques galets limitent des sols en terre battue. Un niveau comportant de nombreux fragments d'amphores Dressel 1 a été mis au jour en limite nord. Ce lit de tessons est actuellement le principal indice d'une occupation laténienne préalable au vicus gallo-romain ${ }^{5}$.

Au sud-est, les chantiers 2 et 5 ont permis l'étude de l'arrière de parcelles bâties et ont livré un abondant matériel antique dispersé dans une colluvion brun foncée. Quelques épandages prennent la forme de larges lentilles composées de petits blocs calcaires et de nombreux fragments de tegulae, de céramiques et de faune. Un fossé
$\mathrm{N} / \mathrm{S}$ légèrement décalé vers l'Ouest et une ligne de poteaux y sont également apparus, témoignant de la présence d'édifices légers, de silos et de dépotoirs. Toutefois, le lien entre ces deux derniers chantiers et l'habitat du secteur B reste ténu, un espace stérile, révélé par le sondage nord du chantier 8 , les séparant.

Enfin, la partie orientale du secteur B se distingue par la découverte hors contexte de fragments d'inscriptions. Sur le chantier 1 , à proximité de la voie, des prospections anciennes ont livré une plaque de bronze dédiée à un notable viennois ayant exercé des charges de duumvir et de triumvir (Béal, Carrara 1997; ILN, Vienne 566). Les déblais des travaux du chantier 2 ont livré une suspension en forme de fer à cheval, élément d'une offrande à une déesse inconnue d'origine locale dénommée "Coriotana" (Carrara et al. 2000 ; ILN, Vienne 565). Cette "concentration" toute relative a nourri l'hypothèse de l'existence d'un monument votif dans ce secteur, éventuellement situé sous l'église. Au moins deux arguments vont pourtant à l'encontre de cette idée. Il faut d'abord signaler que la plaque commémorative a été découpée et n'a donc pas été trouvée in situ. De plus tous les sondages réalisés sur la Place de l'église, autour et même en partie sous le sanctuaire médiéval, se sont révélés stériles non seulement en vestiges mais aussi en matériels antiques.

\subsection{Les marges de l'occupation}

Plusieurs opérations de sondages ont porté sur les marges de l'occupation antique (fig. 5).

Au sud, les chantiers 8,10 et 12 n'ont livré aucun matériel gallo-romain malgré la proximité de parcelles densément occupées (chantiers 2 et 5). De même au sudouest, aucun témoin d'habitat n'a été trouvé dans le chantier 11. Les importantes concentrations de tessons trouvées en prospection sur les parcelles A265, 266 et 267 semblent provenir de fosses dépotoirs creusées en limite de site et ne paraissent pas signaler l'existence en ce lieu d'un habitat ou d'ateliers.

L'extension au Nord est plus facile à cerner. Le chantier 9 n'a pratiquement pas livré de matériel ancien alors que, immédiatement au sud, le chantier du Syndicat des Eaux (chantier 0) a fourni un important mobilier et des traces de constructions.

La limite orientale reste mal connue. Le secteur C révèle des zones d'occupations peu denses. On y note plusieurs fonds de fosses indéterminées avec un peu de matériel céramique (chantier $n^{\circ} 3$ ) ou un large enclos palissadé (fig. 8) orienté Nord-Sud ainsi qu'un empierrement (site 6). Peut-être est on déjà, à cet endroit, face à un établissement rural situé en bordure de l'agglomération.

\footnotetext{
5 La mise en place "sauvage" des réseaux de viabilisation de deux villas occupant l'emprise de l'habitat a irrémédiablement perturbé cette zone. Le relevé des coupes des tranchées a uniquement permis de repérer d'autres fondations et fosses.
} 




Fig. 9. La villa du Paradis d'après la prospection pédestre.

\section{SYNTHÈSE}

Les opérations d'archéologie préventive engagées ces dernières années n'ont pas encore fourni suffisamment d'informations pour comprendre l'organisation de l'espace dans l'agglomération antique d'Optevoz. Elles permettent malgré tout d'ores et déjà d'en appréhender l'extension. Sans être un village-rue, l'urbanisation se développe autour d'un nœud routier constitué d'une voie de communication avec la vallée du Rhône et d'un ou deux chemins de desserte traversant le plateau. Cette organisation en trois branches distingue très nettement Optevoz des autres sites de l'Isle-Crémieu qui, dans le cas des grandes villae du Vernai à Saint-Romain-de-Jalionas, Cozance à Trept ou Charnioux à Saint-Baudille-de-laTour (fig. 2), peuvent couvrir des surfaces similaires. Même si aucune inscription ne permet à ce jour d'en préciser le nom ou le statut, il n'est pas douteux qu'Optevoz soit le seul habitat groupé reconnu sur le plateau à ce jour.

L'origine et la chronologie du site posent encore question. Une occupation laténienne a été très ponctuellement mise en évidence mais il n'est pas certain qu'il s'agisse déjà à cette époque d'un village ni que l'agglomération antique lui succède sans hiatus. Celle-ci paraît perdurer pendant les trois premiers siècles de notre ère, les témoins d'occupation du $I^{\mathrm{e}} \mathrm{s}$. se faisant beaucoup plus rares. Il n'est pas encore possible d'assurcr quc l'assiette du site soit restée inchangée au cours de ces quatre siècles. De même, il reste à vérifier la réalité de la continuité d'occupation qui permettrait de relier l'habitat du Bas-Empire et le village médiéval dont les premiers vestiges retrouvés remontent seulement au $\mathrm{X}^{\mathrm{e}}$ siècle.
L'organisation de l'espace dans cette agglomération reste méconnue. Les différences constatées dans les orientations des constructions partiellement dégagées permettent d'exclure tout schéma d'organisation orthonormé préétabli. On peut se demander si l'absence de traces d'une architecture monumentale est uniquement liée à une lacune de la recherche ou si, au contraire, elle n'est pas révélatrice d'une réalité antique. Aucun indice de thermes n'a jamais été relevé. Le catalogue épigraphique est pauvre et il n'est pas certain que l'objet votif dédié à Coriotana ait été déposé dans un lieu de culte public. Seule l'inscription au duumvir, trouvée hors contexte, suggère une évergésie ou un monument commémoratif. $\mathrm{La}$ concentration de stèles funéraires et une information orale faisant état de la découverte de tombes antiques autour du chantier 2 sont autant d'indices qui, par contre, indiqueraient un cimetière antique sous la partie sud-est du village.

L'essentiel des données fournies par les évaluations concerne l'habitat mais, là encore, il n'a pas été possible d'en dresser un plan. La présence d'activités domestiques (mouture, tissage), est attestée partout mais la question des métiers et des productions de ses habitants doit être évoquée. La multiplicité des foyers trouvés dans le chantier 7 incite à restituer des activités artisanales encore mal déterminées même si des traces éparses de métallurgie du fer et du bronze ont été retrouvées. Faute de ressources locales en minerai, il est néanmoins douteux que cet artisanat ait été développé à grande échelle. Une partie notable de la population devait se consacrer à des activités rurales. La carte archéologique ne montre aucune implantation agricole notable dans un vaste rayon autour de l'agglomération. Tout au plus peuton signaler à l'est et au sud un réseau de fermes implantées en périphérie de la doline (fig. 4, sites 5, 7, 11, 17 , 18). Les chantiers 3 et 6 (fig. 5) ont fourni des enclos et des fosses pouvant signaler la proximité d'exploitations agricoles. Il convient de noter l'absence de villae dans la doline qui concentre les terrains les plus favorables à la culture. Plusieurs grandes villae (fig. 4 , sites $2,16,20$ ) ont pourtant été découvertes à deux ou trois kilomètres de l'agglomération mais semblent plutôt consacrées à l'exploitation de vallées humides qu'à la mise en valeur de la doline dont elles sont topographiquement séparées par des buttes. Seule la villa du Paradis (site 1), située à $700 \mathrm{~m}$ à l'ouest de l'agglomération, entretient visiblement une relation avec elle. Les découvertes qui se sont accumulées depuis le XIX ${ }^{\mathrm{e}}$ siècle sur cet établissement permettent de le classer parmi les plus riches de l'Isle-Crémieu malgré sa taille relativement réduite (fig. 9). La morphologie de cet établissement dessinée par plusieurs années de prospections fines (Carrara 2000) est pourtant anormale. Au vu des ramassages de surface, la pars rustica ne couvre qu'environ $7000 \mathrm{~m}^{2}$, soit guère plus que la pars urbana 
qui est, elle, une des plus vastes des villae du secteur. Même si, bien évidemment, la partie économique est moins facilement lisible en prospection que la partie résidentielle, il ne semble pas que ce rapport soit seulement imputable à de mauvaises conditions d'observation et notamment à un recouvrement différentiel. En effet, la villa n'est pas implantée immédiatement au pied de la falaise et d'hypothétiques colluvions auraient d'abord recouvert soit la partie résidentielle, plus proche, soit la cuvette marécageuse qui représente le point le plus bas de cette zone. Cet équilibre entre les parties économiques et résidentielles n'est pas courant dans les villae prospectées dans l'Isle Crémieu et ses marges où on constate habituellement un rapport oscillant entre $1 / 3$ et $1 / 6$. On peut donc envisager que l'agglomération d'Optevoz ait pu accueillir une partie de la population artisanale et paysanne de la villa. Ce qui vicnt d'être évoqué pour les installations de la villa se pose aussi pour le foncier. Il n'est guère pensable que le domaine de cette exploitation agricole se soit limité à la cinquantaine d'hectares chaotiques du piémont de falaise au pied de laquelle elle est implantée. S'ils n'entretenaient peut-être pas un rapport de dépendance aussi étroit que celui constaté entre le vicus de Bliesbruck et la villa de Reinheim (Petit 2000) on peut vraisemblablement restituer au moins un lien économique entre l'agglomération d'Optevoz et la villa du Paradis. Il est tentant d'envisager, même s'il est impossible de le prouver, qu'un des propriétaires de la villa puisse ainsi avoir été ce notable viennois anonyme dont le souvenir est commémoré sur la plaque trouvée dans le village. Il est à noter que l'Isle-Crémieu compte plusieurs exemples de ces grands aristocrates ayant cumulé les charges en ville mais possédant de grandes résidences à la campagne.
Optevoz se comprend moins par rapport à une hypothétique intégration dans un réseau de voies de communication à longue distance que dans le cadre de la mise en valeur d'un petit bassin cultivable et de ses marges. À Optevoz, comme partout ailleurs dans l'Isle-Crémieu, la villa reste l'échelon essentiel dans l'organisation de l'espace rural et l'encadrement de la population. La carte archéologique de l'Isle-Crémieu (fig. 2) montre ainsi la grande similitude de densité de sites importants dans la vallée du Rhône et dans les parties labourées et prospectées du plateau.

\section{CONCLUSION}

Le croisement des données de prospection et des sondages a permis de reconsidérer l'importance réelle de l'agglomération d'Optevoz. Comme pour d'autres sites de la région (Royet, Faucher 2002), il est évident qu'une confusion entre le site et le territoire de la commune où il est implanté a conduit à le surévaluer.

Il faut encore approfondir la relation qui se dessine entre la villa du Paradis, l'agglomération et le petit finage qu'ils exploitent. L'agglomération antique sous Optevoz reste encore largement à caractériser. On peut s'interroger pour savoir si une approche s'appuyant uniquement sur des évaluations permettra encore de progresser dans cette recherche. Une approche plus extensive des vestiges par la fouille semble indispensable pour enfin appréhender leur organisation.

R. ROYER - Conservateur du patrimoine, SRA Rhône-Alpes.

C. CoQuiDÉ - Chargée d'étude et de recherche, INRAP RhôneAlpes/Auvergne.

\section{BIBLIOGRAPHIE}

\section{Ouvruges publiés}

Béal, Carrara 1997 : BÉAL (J.-C.), CARRARA (S.) - Une inscription inédite d'Optevoz (Isère, France). Zeitschrift für Papyrologie und Epigraphik, 119, 1997, pp. 213-216.

Boucher, Tassinari 1976 : BOUCHER (S.), TASSINARI (S.) Bronzes antiques du musée de la Civilisation gallo-romaine de Lyon: inscriptions, statuaire, vaisselle, tome 1. Lyon, 1976.

Buisson, Pelletier 1983 : BUISSON (A.), PELLETIER (A.) - Les voies romaines autour de Lyon. Essai de synthèse. In: CHEVALLIER (R.) dir. - Les voies anciennes en Gaule. Actes du colloque Caesarodunum $n^{\circ}$ XVIII. Paris, 1983, pp. 157-166.

Carrara, Coquidé, Guyon 2000 : CARRARA (S.), COQUIDÉ (C.), GUYON (M.) - Une dédicace inédite à la déesse Coriotana à Optevoz (F, Isère). Instrumentum $n^{\circ} 12,2000$, pp. 16-17.

Chagny 1929 : CHAGNY (A.) - Sites et monuments de la région de Crémieu. Lyon, 1929.

Chauffin 1960 : CHAUFFIN (J.) - Les stations gallo-romaines du Bas Dauphiné. Évocations, 1960, pp. 93-96.
Chauffin 1981 : CHAUFFIN (J.) - Vingt ans après un premier inventaire de nos sites gallo-romains. Évocations, 1981, pp. 1-10.

Collectif 1987 : Histoire des communes de l'Isère, Arrondissement de la Tour-du-Pin. Horvath, Lyon, 1987.

Commarmond 1855 : COMMARMOND (A.) - Description des Antiquités et Objets d'art des salles des arts de la ville de Lyon. Lyon, 1855.

Loriot, Rémy 1988 : LORIOT (X.), RÉMY (B.) - Corpus des trésors monétaires antiques de la France. Rhône-Alpes, $t$. V. Paris, 1988.

Michel 1987 : MICHEL (J.-C.) - Isère gallo-romaine, $2^{e}$ partie: arrondissements de la Tour du Pin et de Vienne. Grenoble, 1987.

Müller 1931 : MÜLLER (H.) - Mobilier d'une sépulture gauloise découverte près de Crémieu. Bulletin de la Société Dauphinoise d'Ethnologie et d'Archéologie, t. 28, 1931, pp. 6-7.

Perrin, Schöenfelder 2003 : PERRIN (F.), SCHÖENFELDER (M.) dir. - La tombe à char de Verna (Isère). ALPARA, Lyon, 2003 (DARA n $\left.{ }^{\circ} 24\right)$. 
Petit 2000 : PETIT (J.-P.) dir. - Le complexe des thermes de Bliesbruck (Moselle). Un quartier public au cour d'une agglomération secondaire de la Gaule Belgique. Paris, éd. Errance, 2000 (BLESA, 3).

Porte 1981 : PORTE (P.) - Le terroir de Hières sur Amby. In: Catalogue de l'exposition Des Burgondes à Bayard. Mille ans de Moyen Âge. Grenoble, Lyon, Genève, 1981, pp. 33-34.

Rémy 2000 : RÉMY (B.) - À propos du Rhône comme limite de la cité de Vienne au Haut-Empire (en amont de Lyon). RANarb, 33, 2000 , pp. 55-61.

Royet, Faucher 2002 : ROYET (R.), FAUCHER (F.) - La villa de Sainte-Colombe et le soit- disant vicus de Travers à La-Balme-lesGrottes (Isère). Réinterprétation des données archéologiques. RANarb, 35, 2002, pp. 279-291.

\section{Recueils d'inscriptions}

ILN, Vienne: RÉMY (B.) dir., BERTRANDY (Fr.), KAYSER (Fr.) PELLETIER (A.), WIBLÉ (A.) - Inscriptions Latines de Narbonnaise (ILN), V, 2, Vienne. Paris, CNRS éd., 2004 (suppl. à Gallia, XLIV).

CIL XII : HIRSCHFELD (O.) - Corpus Inscriptionum Latinarum (CIL), vol. XII. Berlin, 1886.

\section{Rapports et diplômes universitaires}

Bleu 2003 : BLEU (S.) - Optevoz "Les Verchères". Rapport d'évaluation multigraphié. INRAP, Lyon, 2003.

Carrara 2000 : CARRARA (S.) - Recherche sur l'occupation antique du plateau de l'Isle Crémieu: les communes de Saint Baudille de La Tour et Optevoz. Mémoire de maîtrise multigraphié. Université de Lyon II, 2000, 2 vol.

Coquidé 1998 : COQUIDÉ (C.) - Optevoz (Isère), "Au Champ". Rapport d'évaluation multigraphié. SRA Rhône-Alpes, Lyon, 1998.

Coquidé, Bonnet, Joly 2004 : COQUIDÉ (C.), BONNET (C.), JOLY (J.-L.) - Optevoz (Isère), "Croix Batailler-École". Rapport de diagnostic d'archéologie préventive. INRAP, Lyon, 2004.
Coquidé, Brouillaud 1997 : COQUIDÉ (C.), BROUILLAUD (S.) Optevoz (Isère), "Champ-Batailler". Rapport d'évaluation multigraphié. SRA Rhône-Alpes, Lyon, 1997.

Coquidé, Faletto 2000 : COQUIDÉ (C.), FALETTO (J.) - Optevoz (Isère), "Au Champ-parcelle 15I". Rapport d'évaluation multigraphié. SRA Rhône-Alpes, Lyon, 2000.

Coquidé, Gianola 1999 : COQUIDÉ (C.), GIANOLA (E.) - Optevoz (Isère), "Clos Batailler". Rapport d'évaluation multigraphié. SRA Rhône-Alpes, Lyon, 1999.

Coquidé, Guyon 1999 : COQUIDÉ (C.), GUYON (M.) - Optevoz (Isère), "Auberges des peintres". Rapport d'évaluation multigraphié. SRA Rhône-Alpes, Lyon, 1999.

Coquidé, Kovatchevitch 1998 : COQUIDÉ (C.), KOVATCHEVITCH (C.) - Optevoz (Isère), "Au champ". Rapport d'évaluation multigraphié. SRA Rhône-Alpes, Lyon, 1998.

Coquidé, Lalaï 2003a: COQUIDÉ (C.), LALAÏ (D.) - Optevoz (Isère), "Propriété Chalard". Rapport d'évaluation multigraphié. INRAP, Lyon, 2003.

Coquidé, Lalaï 2003b : COQUIDÉ (C.), LALAÏ (D.) - Optevoz (Isère), "Chemin de la Cure-Batailler". Rapport d'évaluation multigraphié. INRAP, Lyon, 2003.

Coquidé, Poole 2002 : COQUIDÉ (C.), POOLE (M.) - Optevoz (Isère), "Croix Batailler - parcelles 392/394". Rapport d'évaluation multigraphié. INRAP, Lyon, 2002.

Coquidé, Savino 2003 : COQUIDÉ (C.), SAVINO (V.) - Optevoz (Isère), "Trio-Batailler". Rapport d'évaluation multigraphié. INRAP, Lyon, 2003.

Coquidé, Franc, Lalaï 2003 : COQUIDÉ (C.), FRANC (O.), LALAÏ (D.) - Optevoz (Isère), Clos du Château-Batailler. Rapport d'évaluation multigraphié. INRAP, Lyon, 2003.

Nourrissat 1999 : NOURRISSAT (S.) - Optevoz (Isère), "Propriété Charvin". Rapport d'évaluation multigraphié. SRA Rhône-Alpes, Lyon, 1999. 